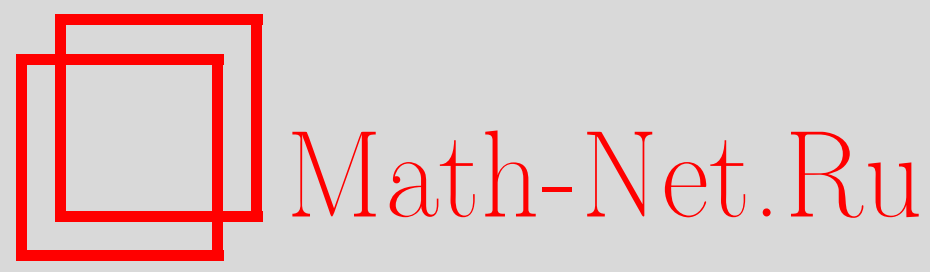

А. А. Тужилин, Минимальные бинарные деревья с правильной границей: случай скелетов с пятью концами, $M a-$ тем. заметки, 1997, том 61, выпуск 6, 907-921

DOI: https://doi.org/10.4213/mzm1574

Использование Общероссийского математического портала Math-Net.Ru подразумевает, что вы прочитали и согласны с пользовательским соглашением http://www . mathnet.ru/rus/agreement

Параметры загрузки:

IP: 54.162 .27 .143

26 апреля 2023 г., $04: 27: 38$

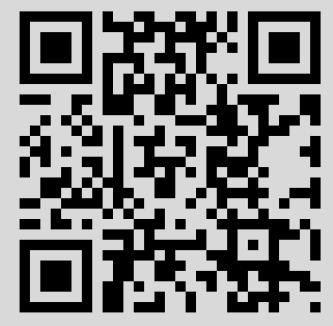




\section{МИНИМАЛЬНЫЕ БИНАРНЫЕ ДЕРЕВЬЯ \\ С ПРАВИЛЬНОЙ ГРАНИЦЕЙ: \\ СЛУЧАЙ СКЕЛЕТОВ С ПЯТЬЮ КОНЦАМИ}

\section{А. А. Тужилин}

В работе изучаются локально минимальные бинарные деревья, затягивающие вершины правильных многоугольников. Описание дается на двойственном языке - языке диагональных триангулящий многоугольников. Рассматриваются диагональные триангуляции специального вида, называемые скелетами. Показывается, что плоские бинарные деревья, двойственные к скелетам с пятью концами, не встречаются среди локально минимальных бинарных деревьев, затягивающих вершины правильных многоугольников.

Библиографоия: 14 названий.

1. Введение. В настоящей статье мы продолжаем исследование локально минимальных плоских сетей, затягивающих вершины правильных многоугольников, начатое в [1] и [2]. Напомним, что локально минимальные сети являются обобщением абсолютно минимальных деревьев, возникающих при решении знаменитой проблемы Ферма-Ярника-Кесслера.

ЗАДАчА. Среди всех сетей (одномерных континуумов), затягивающих данное конечное множество точек плоскости, назьваемое граничньм множеством, найти сеть наименьшей длины.

Эта задача обычно называется проблемой Штейнера, в честь швейцарского ученого Якоба Штейнера (1796-1863). Однако, как было отмечено в [3], простейший вариант этой проблемы для случая, когда граничное множество состоит из трех точек, возник еще в работах Ферма (1601-1665). В современном виде проблема была сформулирована Ярником и Кесслером в 1934 году [4]. Само же название - проблема Штейнера укоренилось благодаря популярности замечательной книги Куранта и Роббинса “Что такое математика", в которой случай Ферма был назван проблемой Штейнера, а проблема Ярника и Кесслера формулировалась как обобщение проблемы Штейнера. Из-за возникшей путаницы сегодня подавляющее большинство специалистов пользуется терминологией, связанной с именем Штейнера.

Работа выполнена при частичной поддержке Национального Центра научных исследований Франции, Международного научного фонда, Российского фонда фундаментальных исследований и Комитета по развитию науки, технологии и экономики (FAPESP) штата Сан Пауло (Бразилия), грант № 93/0248-7. Работа в рамках последнего гранта проходила в Математическом институте (IMECC) университета штата Сан Пауло в Кампинасе (UNICAMP). 
Хорошо известна локальная структура локально минимальных сетей, в частности, абсолютно минимальных деревьев. Напомним, что каждая такая сеть составлена из отрезков - ребер сети, стыкуюшихся в вершинах сети под углами не менњше, чем $120^{\circ}$. В частности, в одной вершине не может встречаться более трех отрезков (степень каждой вершины непревосходит 3). Кроме того, в случае локально минимальных бинарных деревьев известен знаменитый алгоритм Мелзака [5], позволяющий определить: можно ли данное граничное множество затянуть локально минимальной сетью данной топологии; и если да, то построить эту сеть.

Учитывая тот факт, что сеть наименьшей длины является деревом и каждое плоское дерево, степени вершин которого не превосходят 3, может быть представлено в виде объединения бинарных деревьев (назьваемых невырожденньми компонентами), стькуюшихся в вершинах степени 2 исходного дерева, можно (перебирая всевозможные топологии таких деревьев, запуская для каждой невырожденной компоненты выбранного дерева алгоритм Мелзака, вычисляя, в случае удачи, длину полученной сети и выбирая из построенных сетей дерево наименьшей длины) построить, в конце концов, абсолютно минимальное дерево. И только что описанный грубьй алгоритм, и более тонкие алгоритмы оказьваются NP-полньпи [6], [7]. Значит, скорее всего, не существует полиномиального алгоритма, решающего проблему Ферма-Ярника-Кесслера. Отметим, что современные компьютеры могут эффективно строить абсолютно минимальные деревья, лишь когда граничное множество содержит не более 30 точек, что достаточно мало для практически значимых задач [7]. Поэтому возникает потребность в поиске геометрических свойств граничных множеств, которые позволяли бы a priori отсеять большее количество возможных топологий сетей.

В предыдущих статьях [1], [2], [8]-[11] А. О. Иванов и А. А. Тужилин построили полное описание локально минимальных бинарных деревьев с выпуклой границей и применили созданную теорию к случаю, когда граница - множество вершин правильного многоугольника (в дальнейшем называемая правильной границей). Чтобы сформулировать основной результат данной работы, дадим следующие определения.

Сетью мы называем связное подмножество евклидовой плоскости $\mathbb{R}^{2}$, представимое в виде плоского графа, ребра которого - гладкие регулярные кривые. Длиной сети назьвается сумма длин всех ребер этой сети. Ясно, что длина сети не зависит от ее представления плоским графом. В дальнейшем, формулируя различные результаты, касающиеся сетей, будем предполагать, не оговаривая каждьй раз, что эти результаты имеют место для некоторого представления сети в виде плоского графа.

Фиксируем некоторое конечное множество $M$ точек плоскости $\mathbb{R}^{2}$. Мы говорим, что сеть Г затягивает $M$, или $M$ является граниией сети $\Gamma$, если $M$ - подмножество множества вершин из Г. Сеть, имеющая наименьшую длину среди всех сетей, затягивающих множество $M$, называется абсолютно минимальной сетью с әраницей $M$. Сеть Г назьвается локально минимальной сетью с әраницей $\partial Г$, если каждая точка $P$ из $Г$ обладает такой замкнутой окрестностью $U$, что пересечение $U \cap \Gamma$ является абсолютно минимальной сетью с границей $(U \cap \partial \Gamma) \cup(\partial U \cap \Gamma)$. Как отмечалось вьше, абсолютно минимальные сети являются также и локально минимальными. Так как мы собираемся изучать только локально минимальные сети, то в дальнейшем, для краткости, будем, как правило, называть локально минимальные сети просто минимальньми.

Следующее предложение полностью описьвает локальную структуру минимальных сетей. 
ПРЕДЛОЖЕНИЕ 1.1. Сеть Г с границей $M$ минимальна, если и только если для одного из ее представлений имеют место следующие свойства:

1) все ребра сети - прямолинейные отрезки;

2) угол межху любыми двумя смежными ребрами не меньше $120^{\circ}$;

3) все вериины степени 1 и 2 являются граничными, т.е. принадлежат $M$.

В настоящей работе рассматриваются только минимальные сети, являющиеся деревьями и не имеющие вершин степени 2. Из предложения 1.1 следует, что степени вершин таких сетей равны 1 или 3 , т.е. эти сети являются бинарньми деревьями (или, иначе, 2-деревьями). Более того, все ребра таких сетей - прямолинейные отрезки, встречаюшиеся в вершинах степени 3 под равными углами величины $120^{\circ}$.

Кроме того, нас будут особенно интересовать минимальные 2-деревья, имеющие выпуклую и, в частности, правильную границу. Напомним, что граничное множество $M$ сети Г называется выпуклым (правильным), если $M$ - множество вершин выпуклого (правильного) многоугольника. Легко показать, что вершины степени 3 минимального дерева с выпуклой границей не являются граничньми. В частности, у минимального бинарного дерева, затягивающего вершины выпуклого многоугольника, граница состоит в точности из всех вершин степени 1 .

В соответствии со сделанньги замечаниями для любого 2-дерева $Г$ (плоского или минимального) вершины степени 1 будем назьвать граничны.ми, а вершины степени 3 внутренними. Ребро из $Г$, инцидентное граничной вершине, будем также называть граничным, а ребро, не инцидентное никакой граничной вершине, - внутренним.

Пусть Г- произвольная сеть. Мы говорим, что Г имеет минимальную реализацию, если существует минимальная сеть $\Gamma_{m}$, планарно эквивалентная $\Gamma$. Если при этом можно найти минимальную сеть $\Gamma_{m}$ с вьпуклой (соответственно правильной) границей, то будем говорить, что Г имеет выпуклую минимальную реализацию (правильную минимальную реализачию), или СМ-реализачию (RM-реализачию).

Оказьвается, исследование локально минимальных бинарных деревьев, особенно в случае выпуклых границ, чрезвычайно удобно проводить на двойственном языке, объектами которого являются диагональные триангуляции многоугольников. Напомним, что по каждому плоскому бинарному дереву можно построить естественнњм образом триангулящию диагоналями некоторого (например, выпуклого или правильного) многоугольника. Такие триангуляции мы назьваем диагональными. Обратно, двойственньй граф произвольной диагональной триангуляции является плоским бинарным деревом. Треугольники таких триангуляций будем назьвать ячейками. (Точные определения см., например, в [12].)

Пусть $T$ - произвольная диагональная триангулящия многоугольника $W$ и $\Delta$ - любая ее ячейка. Ячейку $\Delta$ назовем крайней, если, по меньшей мере, две ее стороны являются сторонами из $W$. Ячейка $\Delta$ называется внутренней, если ни одна из ее сторон не является стороной из $W$. Если две ячейки пересекаются по стороне, то мы называем их смежсными и говорим, что одна из них примыкает к другой. Крайняя ячейка, примыкающая к внутренней, называется наростом, а триангуляция $T$ без наростов - скелетом. Каждую триангулящию $T$ можно разложить на скелет и наросты с помощью следующего правила: для каждой внутренней ячейки триангуляции $T$ из всех примыкающих к ней наростов, если они есть, надо выбросить ровно один. Легко проверить, что полученная триангулящия $T^{\prime}$ уже не будет содержать наростов, т.е. $T^{\prime}$ является ске- 
летом, которьй называется скелетом триангулящии T. Отметим, что построенное разложение, вообще говоря, неоднозначно.

Мы говорим, что Т имеет выпуклую минимальную реализацию (правильную минимальную реализачию), если ее двойственньй граф обладает такой реализацией. В [1], [8]-[11] для триангулящий $T$, обладающих выпуклой минимальной реализацией, были описаны все скелеты (оказалось, что они устроены достаточно просто), а также способы крепления к ним наростов, при которых полученные триангуляции по-прежнему будут обладать вьпуклой минимальной реализацией. Интересным следствием из [1], [8]-[11] является тот факт, что скелет, обладающий выпуклой минимальной реализацией, содержит не более шести крайних ячеек, и значит, не более четырех внутренних ячеек. Кроме того, в [1] как следствие общей теории были описаны все скелеты с не более чем тремя крайними ячейками, обладающие правильной минимальной реализацией. В [2] было показано, что скелеты с четырьмя крайними ячейками не имеют правильной минимальной реализации.

В настоящей работе воспользуемся техникой, разработанной в [1] и [2], для доказательства следующей теоремы.

ОСНовНАЯ ТЕОРЕМА. Скелеты с пятью крайними ячейками не имеют правильной минимальной реализачии.

Автор благодарен академику РАН профессору А. Т. Фоменко за постоянное внимание к работе, а также к.ф.-м.н. А. О. Иванову за многолетнее плодотворное сотрудничество.

2. Предварительные результаты. Приведем основные результаты, полученные в [1], [2] и [8]-[11].

Для изучения минимальных 2-деревьев очень полезньм оказалось понятие числа вращения, введенное А. О. Ивановым и А.А. Тужилиньп в [8]. Мы приведем наглядное неформальное определение числа врашения (формальное определение см. в [8]).

Рассмотрим произвольное плоское 2-дерево $\Gamma$, выберем некоторую пару $(a, b)$ его ребер. Пусть $\gamma$ - единственньй путь в $\Gamma$, соединяющий эти ребра. При “движении" по пути $\gamma$ от ребра $a$ к ребру $b$ в каждой внутренней вершине из $\gamma$ мы “поворачиваем" или налево, или направо. Разность числа левых и правых поворотов во всех внутренних вершинах пути $\gamma$ назьвается числом вращения упорядоченной пары $(a, b)$ и обозначается tw $(a, b)$. Числом вращения tw $Г$ бинарного дерева Г называется максимум чисел врашения $\mathrm{tw}(a, b)$, взятьй по всевозможным упорядоченным парам ребер из $\Gamma$.

ПРЕДЛОЖЕНИЕ 2.1. Плоское 2-дерево Г имеет выпуклую минимальную реализацию тогда и только тогда, когда tw $\Gamma \leqslant 5$.

ЗАмЕчАниЕ. Результат предложения 2.1 может быть обобщен как на случай минимальных 2-деревьев с произвольной границей, так и на случай взвешенных минимальных 2-деревьев (см. [13], [14]).

Мы уже говорили, что плоские 2-деревья могут быть описаны на двойственном языке, а именно, на языке диагональных триангулящий плоских многоугольников: по каждой диагональной триангуляции $T$ многоугольника $W$ можно построить двойственную сеть $\Gamma_{T}$, являющуюся плоским бинарньм деревом, и обратно, по каждому плоскому бинарному дереву Г можно построить диагональную триангулящию некоторого плоского многоугольника $W$ такую, что двойственная сеть $\Gamma_{T}$ этой триангуляции планарно эквивалентна Г (подробнее, см. в [12]). 
Введем следующее соглашение: если двойственная сеть диагональной триангуляции $T$ обладает некоторым свойством, то мы будем говорить, что сама триангуляция $T$ обладает этим свойством. Так, например, совокупность ячеек из $T$ назьвается связной, если пересечение двойственной сети $\Gamma_{T}$ с этими ячейками связно. Будем говорить, что диагональная триангуляция $T$ имеет минимальную реализацию (соответственно выпуклую минимальную реализачию, правильную минимальную реализачию), если такой реализацией обладает ее двойственная сеть.

Дадим теперь описание диагональных триангулящй многоугольников $W$, имеющих выпуклую минимальную реализацию. В соответствии с предложением 2.1 диагональная триангулящия $T$ обладает выпуклой минимальной реализацией, если и только если число вращения двойственной сети $\Gamma_{T}$ не превосходит 5 . Класс таких диагональных триангуляций традиционно обозначаем через $\mathscr{W} \mathscr{P}_{5}$. Для удобства изложения, ограничимся рассмотрением лишь тех многоугольников $W$, которые содержат не менее пяти вершин (случаи треугольника и четырехугольника очевидны). Таким образом, каждая изучаемая нами диагональная триангуляция содержит не менее трех ячеек.

Во введении описаны некоторые структурные элементы диагональных триангуляций, в частности, разложение диагональной триангуляции на скелет и наросты. Для формулировки основной классификационной теоремы, описывающей все диагональные триангулящии, имеющие вьпуклую минимальную реализацию, понадобятся также следуюшие понятия.

Пусть $T$-произвольная диагональная триангулящия многоугольника $W, T=S \cup\left\{\Delta_{i}\right\}$ - разложение триангуляции $T$ на скелет $S$ и наросты $\Delta_{i}$. Связные компоненты множества внутренних ячеек скелета $S$ назьваются узлами ветвления, а связные компоненты скелета $S$, из которого выброшены все внутренние ячейки, назьваются линейны.ми участками. Линейньй участок, содержащий крайнюю ячейку, назьвается концом, а не содержаший - перегородкой. Итак, мы построили разложение произвольной диагональной триангулящии на узлы ветвления, линейные участки и наросты. Вновь отметим, что это разбиение, вообще говоря, неоднозначно.

Следующее предложение полностью описьвает все возможные узлы ветвления скелетов, имеющих вьпуклую минимальную реализацию.

ПРЕДЛОЖЕНИЕ 2.2. Узлы ветвления скелетов, имеющих выпуклую минимальную реализачию, могут быть ровно пяти типов, приведенных на рис. 1.
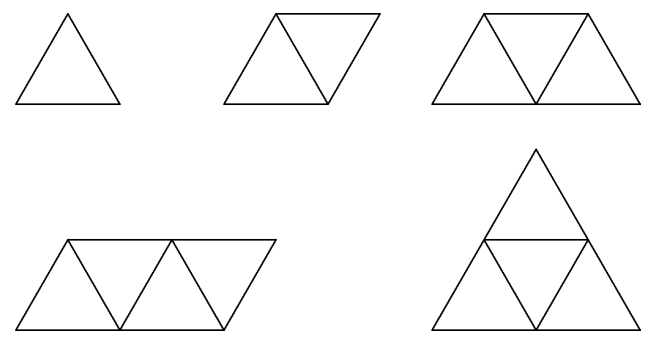

Рис. 1. Узлы ветвления скелетов, имеющих СМ-реализацию

Опишем теперь линейные участки таких скелетов. Чтобы описание было наглядным, полезно рассматривать диагональные триангулящии специального вида, а именно, так 
называемые паркеты. Диагональная триангулящия, все ячейки которой - правильные (конгруэнтные) треугольники, назьвается паркетом. Следующее предложение показьвает, что при изучении минимальных 2-деревьев с вьпуклой границей можно ограничиться паркетами.

ПРЕДЛОЖЕНИЕ 2.3. Каждое плоское 2-дерево с числом вращения, не превосходящим 5, планарно әквивалентно двойственной сети некоторого паркета.

Итак, в дальнейшем всегда будем предполагать, что рассматриваемые диагональные триангулящии являются паркетами.

Пусть $S$ - скелет и $L$ - его линейный участок. Проведем во всех некрайних ячейках скелета $S$ их средние линии, соединяющие внутренние стороны этих ячеек. В крайних ячейках проведем средние линии, параллельные уже построенным в смежных ячейках. Все проведенные средние линии назьваются позвонками. На объединении всех позвонков введем структуру графа, объявив ребрами максимальные связные прямолинейные совокупности позвонков из невнутренних ячеек, а также все позвонки из внутренних ячеек. Полученный плоский граф назьвается позвоночником скелета $S$. Далее пересечение позвоночника скелета $S$ с каждым его линейным участком $L$ называется позвоночником линейного участка $L$.

Следующее предложение [8] описывает все линейные участки скелетов, обладающих СМ-реализацией.

ПРЕДЛОЖЕНИЕ 2.4. Позвоночник каждого линейного участка L скелета, обладающего СМ-реализачией, однозначно проектируется на прямую, параллельную некоторой стороне произвольной ячейки.

Прямая из предложения 2.4 называется направляющей линейного участка L. Отметим, что с точностью до параллельности существует ровно три прямых, каждая из которых параллельна какой-нибудь стороне произвольной ячейки. В дальнейшем, говоря о направляющих, будем отождествлять параллельные прямые, поэтому имеет смысл говорить, что линейньй участок обладает одной направляющей (т.е. все направляющие линейного участка параллельны). Принимая во внимание сделанные замечания и соглашение, отметим, что существует не более трех направляющих. Если линейньй участок обладает тремя направляющими, то он называется змеей, если двумя - лестниией, если одной - ломаной змеей (см. рис. 2). Таким образом, описаны основные структурные элементы паркетов, обладающих СМ-реализацией: наросты, узлы ветвления и линейные участки.

Опишем, как эти элементы крепятся друг к другу. Оказывается, каждый скелет, обладающий СМ-реализацией, можно получить из скелетов специального канонического вида, выбрасьвая из последних некоторое количество ячеек. Операция “выбрасьвания" назьвается редукиией. Различаются редукции I-го и II-го типа.

Пусть $D$ - некоторьй паркет. Редукция I-го типа состоит в разрезании паркета $D$ вдоль некоторой диагонали и отбрасывания одной из двух компонент. Чтобы описать редукцию II-го типа, отметим, что имеется естественное взаимно однозначное соответствие между внутренними ребрами двойственной сети и диагоналями паркета. Итак, редукция II-го типа состоит в следующем: выберем две диагонали паркета $D$ такие, что число врашения между соответствуюшими им внутренними ребрами двойственной сети равно 0; разрежем паркет $D$ вдоль этих диагоналей; выбросим ту из трех образованных компонент, которая пересекается с обеими оставшимися компонентами; с помо- 


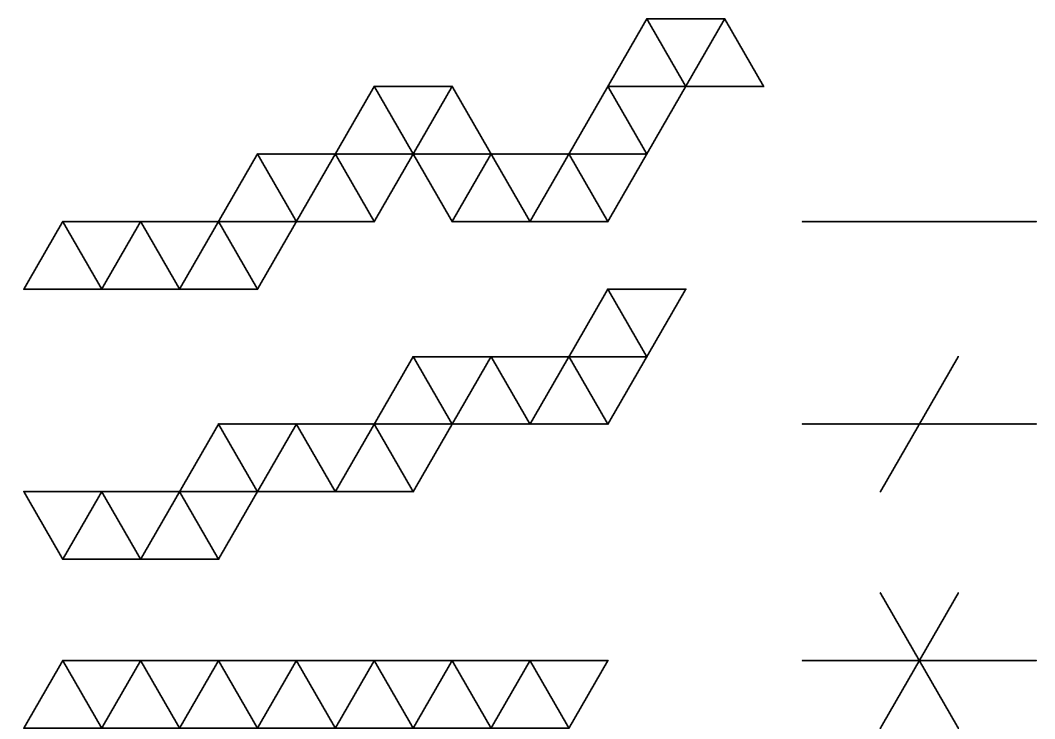

Рис. 2. Линейные участки скелетов, обладающих СМ-реализацией

щью параллельного переноса склеим оставшиеся две компоненты по диагоналям разреза. Можно показать, что обе эти операции не разрушают свойство скелета обладать СМ-реализацией.

Теперь мы можем сформулировать теорему из [8], классифицирующую скелеты, которые обладают СМ-реализацией.

ПРЕДЛОЖЕНИЕ 2.5. Каждый скелет из $\mathscr{W} \mathscr{P}_{5}$ может быть получен кратным применением редукиии к скелету одного из трех канонических типов, схема которых приведена на рис. 3. Три параллельных черточки обозначают ломаную змею, единственная направляющая которой параллельна черточкам; две черточки, пересекаюшиеся под углом $60^{\circ}$, обозначают лестничу с направляющими, параллельными черточкам; одна черточка соответствует змее, позвоночник которой параллелен черточке; и, наконеи, кружсок соответствует узлу ветвления, состоящему из одной ячейки.
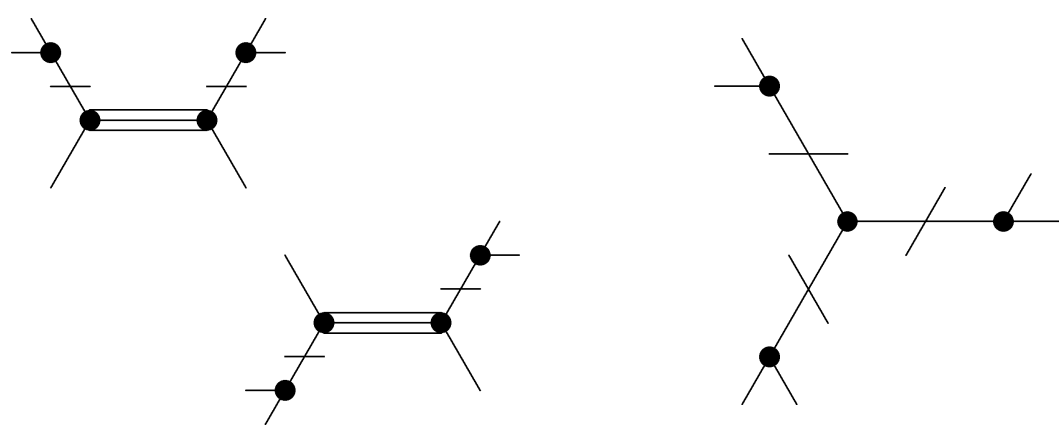

Рис. 3. Схема классифицируюших скелетов 
Из предложения 2.5 вытекает интересное следствие.

СлЕдСтвиЕ 2.1. Скелет из $\mathscr{W} \mathscr{P}_{5}$ содержит не более шести концов, не более трех перегородок и не более четырех внутренних ячеек (а значит, не более четырех узлов ветвления).

Ориентируем позвоночник каждого конща $L$ скелета $S \in \mathscr{W} \mathscr{P}_{5}$ в сторону крайней ячейки, принадлежащей этому концу (для $S$, не содержащего внутренних ячеек, сушествует две таких ориентации). Тогда направления ребер ориентированного таким образом позвоночника назовем направлениями кониа $L$. Ясно, что всего существует не более шести различных направлений, образующих правильный шестиугольник, вписанный в единичную окружность направлений. Такой шестиугольник назовем иестиугольником направлений.

СлЕДСТВИЕ 2.2. Кажддй конеи, скелета $S$ из $\mathscr{W} \mathscr{P}_{5}$ имеет не более трех различных направлений, причем эти направления последовательные. Мнохсества направлений, соответствующих любым двум различным кониам из $S$, не пересекаются.

Если ориентировать граничу скелета $S$, скажем, против часовой стрелки, что задаст ииклический порядок на множестве концов, а также ориентировать шестиугольник направлений против часовой стрелки, что задаст ииклический порядок на семействе множеств направлений концов, то оба возникиих порядка будут согласованы: последовательные концы будут иметь последовательные множества направлений.

Так как мы в дальнейшем будем заниматься только скелетами, то теорему, описывающую возможные расположения наростов на скелетах паркетов из $\mathscr{W} \mathscr{P}_{5}$, приводить не будем, а заинтересованного читателя вновь отсылаем к [1], [8]-[12].

Приведем теперь результаты из [1] и [2], где изучались минимальные 2-деревья с правильной границей, или, иными словами, 2-деревья, обладающие RM-реализацией.

Скелет назьвается линейны.м, если он не содержит узлов ветвления.

ПРЕДЛОЖЕНИЕ 2.6. Линейный скелет из $\mathscr{W} \mathscr{P}_{5}$ имеет RM-реализацию тогда и только тогда, когда он является змеей.

Скелет с тремя кониами имеет RM-реализачию на правильном $п$-угольнике тогда и только тогда, когда $n=6 k+3$, все кониы скелета - змеи, состоящие из одинакового числа ячеек, и скелет инвариантен относительно поворота на $120^{\circ}$ вокруг иентра единственной содержащейся в нем внутренней ячейки.

Скелеты с четырьмя концами не имеют RM-реализачии.

Далее опишем некоторые общие свойства скелетов, допускающих RM-реализацию.

ПРЕДЛОЖЕНИЕ 2.7. Пусть $S$ - произвольный скелет из $\mathscr{W} \mathscr{P}_{5}$, имеющий RM-реализачию. Тогда все концы скелета $S$ - змеи.

Следующее предложение описывает перегородки специального вида.

ПРЕДЛОЖЕНИЕ 2.8. Пусть $S$ - скелет из $\mathscr{P}_{5}$, имеющий не менее четырех кониов и обладающий RM-реализачией. Пусть $Z_{1}$ и $Z_{2}$ - два его кониа-змеи, иниидентные внутренней ячейке $\Delta$ скелета $S$. Предположим, что направления концов $Z_{1}$ и $Z_{2}$ составляют угол $60^{\circ}$. Тогда скелет $S$ не имеет инцидентной $\Delta$ 
перегородки. Иными словами, внутренняя ячейка $\Delta$ входит в состав некоторого узла ветвления, состоящего не менее, чем из двух ячеек.

Далее мы приведем результаты, описывающие возможные длины боковин скелетов, имеющих RM-реализацию. Первые результаты этого типа были получены в [1], а их усиленный вариант, приводимый ниже, - в [2].

Граничная сторона скелета назьвается кониевой, если она пересекается с позвоночником. Замькания связньх компонент границы скелета, из которой выброшены все граничные ребра, называются боковинами скелета. Количество граничных ребер скелета, входящих в боковину, назьвается длиной боковины. Количество всех граничных ребер скелета называется длиной гранииы скелета.

Граничное ребро двойственной сети скелета, приходящее на конщевую сторону, и соответствующее ребро произвольной минимальной реализации этого скелета также назовем кониевыми. Остальные граничные ребра двойственной сети и соответствующей минимальной реализации назовем отростками.

Пусть $S$ - произвольный скелет и $\Gamma$ - некоторая его минимальная реализация, затягивающая множество $M$. Граничную вершину двойственной сети $\Gamma_{S}$, инцидентную концевому ребру, а также соответствующую вершину из $M$, назовем кониевыми.

Везде ниже мы предполагаем, что все рассматриваемые скелеты не являются линейными, т.е. имеют, по крайней мере, одну внутреннюю ячейку, и значит, не менее трех концов.

Пусть $S$ - скелет из $\mathscr{W} \mathscr{P}_{5}$, обладающий RM-реализацией, и $\Gamma=\Gamma_{S}-$ его двойственная сеть. Обозначим через $е$ и $a$ конщевое ребро и смежный с ним отросток 2-дерева $\Gamma$, соответствуюшие некоторому концу-змее $Z$ скелета $S$. Будем рассматривать $e$ и $a$ как векторы, выходящие из общей для них внутренней вершины сети Г. Считая, что плоскость канонически ориентирована, дадим следующее определение.

ОПРЕДЕЛЕНИЕ. Индексом $\operatorname{ind}(Z)$ кониа $Z$ назовем число, равное 1 , если репер $(a, e)$ положительно ориентирован, и $-1-$ в противном случае.

Пусть $Z$ и $Z^{\prime}$ - конщы-змеи скелета $S$.

ОПРЕДЕЛЕНИЕ. Индексом $\operatorname{ind}\left(Z, Z^{\prime}\right)$ упорядоченной пары $\left(Z, Z^{\prime}\right)$ кониов $Z$ и $Z^{\prime}$ назьвается полуразность $\frac{1}{2}\left[\operatorname{ind}\left(Z^{\prime}\right)-\operatorname{ind}(Z)\right]$.

Ясно, что индекс пары концов может принимать лишь три значения: $-1,0$ и 1 . В первом случае говорят, что конщы смотрят наружу, во втором - конщы $с$ мотрят $\boldsymbol{\theta}$ одну сторону, в третьем - концы смотрят внутрь.

Пусть $\left(Z, Z^{\prime}\right)$ - упорядоченная пара конщов-змей скелета $S$. Углом om $Z \kappa Z^{\prime}$ назовем величину дуги единичной окружности направлений, движение по которой от направления первого конца $Z$ к направлению второго конца $Z^{\prime}$ происходит против часовой стрелки, т.е. в положительном направлении.

Пусть $B$ - произвольная боковина скелета $S$. Так как мы предполагаем, что скелет $S$ не является линейным, то существует ровно два конца $Z$ и $Z^{\prime}$, пересекающихся с боковиной $B$. Более того, $B \cap Z$ и $B \cap Z^{\prime}-$ связные фрагменты ломаной $B$, содержащие граничные для $B$ ребра. Будем говорить, что боковина $B$ порождена кониами $Z u$ $Z^{\prime}$, а пересечения $B \cap Z$ и $B \cap Z^{\prime}$ будем назьвать соответственно $Z$ - $u Z^{\prime}$-частями боковины $B$.

Пусть, для определенности, при движении по границе скелета $S$ против часовой стрелки мы проходим боковину $B$, начиная с ее $Z$-части и заканчивая ее $Z^{\prime}$-частью. 
ОпРЕДЕлЕниЕ. В сделанных выше предположениях индекс $\operatorname{ind}\left(Z, Z^{\prime}\right)$ назьвается индексом боковины $B$ и обозначается через ind $(B)$. Угол от конца $Z$ к концу $Z^{\prime}$ назьвается раствором боковины $B$ и обозначается $\operatorname{ang}(B)$.

ПРЕДЛОЖЕНИЕ 2.9. Пусть $S$ - произвольный скелет из $\mathscr{W}_{5}$ и $n$ - длина его гранииы. Предположсим, что $S$ обладает RM-реализачией. Пусть В - произвольная боковина скелета $S, b-$ ее длина $u \operatorname{ang}(B)=\pi l / 3$ - ее раствор. Тогда

1) если $\operatorname{ind}(B)=-1$, то $\mathrm{nl}$ не делится на 6 и $b=[n l / 6]-1$;

2) если $\operatorname{ind}(B)=0$, то остаток $r$ от деления $n l$ на 6 не равен 3 и если $0 \leqslant r \leqslant 2$, mo $b=[n l / 6]-1$; если $4 \leqslant r \leqslant 5$, mo $b=[n l / 6] ;$

3) если ind $(B)=1$, то nl не делится на $6 u$ u $b=[n l / 6]$.

Иными словами, если $n l=6 p+r$, әде $p=[n l / 6], a 0 \leqslant r \leqslant 5$, mо

$\left.1^{\prime}\right)$ при $r=0$ индекс боковины $B$ всегда равен 0, при $r=3$ индекс боковины $B$ никогда не равен 0, а при оставшихся $r$ индекс боковины $B$ мохет быть равен как $0, \operatorname{ma\kappa } u \pm 1$;

$\left.2^{\prime}\right)$ при любом $r>0$ ecлu $\operatorname{ind}(B)=-1$, mo $b=p-1$; $e c \Omega u \operatorname{ind}(B)=1, m o b=p$

$\left.3^{\prime}\right)$ eсли $\operatorname{ind}(B)=0$, mo прu $0 \leqslant r \leqslant 2$ имеем $b=p-1$; при $4 \leqslant r \leqslant 5$ имеем $b=p$.

Пусть $е$ и $e^{\prime}$ - два конщевых ребра скелета $S$. Тогда ломаные, полученныепри замыкании связных компонент, на которые распадается граница скелета $S$ после выбрасывания этих конщевых ребер, назьваются супербоковинами. Пусть $Z$ и $Z^{\prime}-$ конщы скелета $S$ такие, что е $\subset Z$ и $e^{\prime} \subset Z^{\prime}$. Будем говорить, что супербоковина $B$ порождена кониами $Z u Z^{\prime}$, а пересечения $B \cap Z$ и $B \cap Z^{\prime}$ будем называть соответственно $Z$ - $u Z^{\prime}$-частями супербоковины $B$.

Пусть, для определенности, при движении по границе скелета $S$ против часовой стрелки мы проходим супербоковину $B$, начиная с ее $Z$-части и заканчивая ее $Z^{\prime}$-частью.

ОпРЕДЕлЕниЕ. В сделанных выше предположениях, индекс $\operatorname{ind}\left(Z, Z^{\prime}\right)$ называется индексом супербоковины $B$ и обозначается через $\operatorname{ind}(B)$. Угол от конща $Z$ к концу $Z^{\prime}$ назьвается раствором супербоковины $B$ и обозначается $\operatorname{ang}(B)$.

ПРЕДЛОЖЕНИЕ 2.10. Утверждения предложения 2.9 имеют место и для супербоковин.

3. Скелеты с пятью концами. В этом пункте мы докажем основную теорему.

TеОРема 3.1. Скелеты с пятью концами не имеют RM-реализачии.

Начнем с описания некоторых элементарных свойств индексов боковин.

Лемма 3.1. Сумма индексов всех боковин скелета $S$ равна 0.

Две боковины $B$ и $B^{\prime}$ называются соседними, если один из порождающих боковину $B$ концов также является одним из концов, порождающих боковину $B^{\prime}$.

Лемма 3.2. Соседние боковины не могут одновременно иметь индекс 1. 
ЛЕмма 3.3. Соседние боковины не могут одновременно иметь индекс-1.

Пусть $S$ - скелет с пятью концами, имеющий RM-реализацию.

СлЕдСтвиЕ 3.1. У скелета $S$ имеется не более двух боковин индекса 1.

СледСтвИЕ 3.2. У скелета $S$ имеется не более двух боковин индекса -1.

СлЕДСтвИЕ 3.3. Существует ровно три случая:

1) индексы всех боковин скелета $S$ равны 0 ;

2) одна из боковин имеет индекс 1 , одна - индекс-1, остальные три боковины имент индекс 0 ;

3) две боковины имеют индекс 1 , две другие - индекс -1 и ровно одна боковина-индекс 0 .

По-прежнему, $S$ - скелет с пятью концами, обладающий RM-реализацией на правильном $n$-угольнике. Обозначим через $Z_{1}, Z_{2}, Z_{3}, Z_{4}$ и $Z_{5}$ его последовательные концы и предположим, что $Z_{1}$ и $Z_{2}$ выходят из одной внутренней ячейки, $Z_{3}$ и $Z_{4}$ тоже выходят из одной внутренней ячейки. Существует два случая: углы между концами в каждой из пар $\left(Z_{1}, Z_{2}\right)$ и $\left(Z_{3}, Z_{4}\right)$ равны между собой и равны $\pi / 3$; в одной из этих пар, скажем в $\left(Z_{1}, Z_{2}\right)$, угол между концами равен $2 \pi / 3$, в то время как в другой паре угол между концами равен $\pi / 3$.

Рассмотрим первьй случай. Предположим, для определенности, что единственный угол между последовательными концами, которьй может быть равен $2 \pi / 3$, - это угол между $Z_{5}$ и $Z_{1}$. По предложению 2.8 скелет $S$ не имеет перегородок, поэтому с точностью до симметрии он имеет вид, изображенньй на рис. 4.

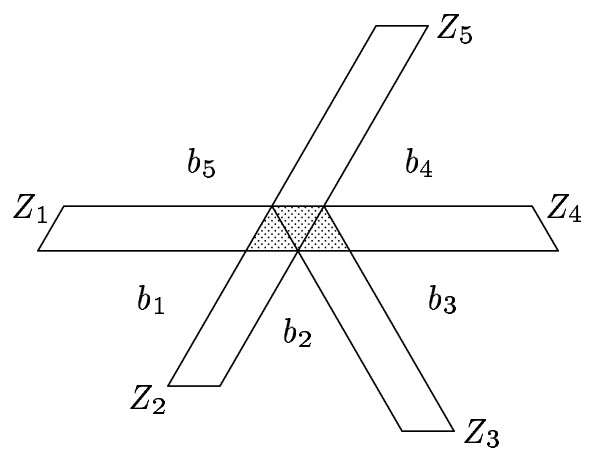

Рис. 4. Такие скелеты не имеют RM-реализации

ПРЕДЛОЖЕНИЕ 3.1. В сделанных выше предположениях скелет $S$, в действительности, не может иметь RM-реализаиии.

ДокАЗАтЕЛЬСтво. Обозначим через $b_{i}$ длину боковины, порожденной конщами $Z_{i}$ и $Z_{i+1}$ (здесь и далее сложение в индексах циклическое, а именно, $\left.i+j=1+(i+j-1) \bmod 5\right)$, и определим вектор $b=\left(b_{1}, \ldots, b_{5}\right)$ длин боковин. Из предложения 2.9 вытекает, что при $i<5 b_{i}=[n / 6]-a_{i}$, где $a_{i}$ равно 0 или 1 , а $b_{5}=[n / 3]-a_{5}$, где $a_{5}$ также равно 0 или 1. Обозначим через $а$ вектор $\left(a_{1}, \ldots, a_{5}\right)$.

Далее, пусть $z_{i}$ обозначает длину конца $Z_{i}$, т.е. количество ячеек, составляющих этот конец. Положим $w_{i}=\left[z_{i} / 2\right]+\left[z_{i+1} / 2\right]$. Пусть $w=\left(w_{1}, \ldots, w_{5}\right)$. Обозначим через $e_{i}$ 
функцию четности конца $Z_{i}$, а именно: $e_{i}$ равно 0 , если $z_{i}$ четно, и равно 1 в противном случае. Теперь все готово, чтобы записать соотношение между длинами концов и боковин скелета $S$. Непосредственные вычисления дают следующий результат:

$$
\begin{aligned}
& b_{1}=\left[\frac{n}{6}\right]-a_{1}=\left[\frac{z_{1}}{2}\right]+\left[\frac{z_{2}}{2}\right]=w_{1}, \\
& b_{2}=\left[\frac{n}{6}\right]-a_{2}=\left[\frac{z_{2}}{2}\right]+\left[\frac{z_{3}}{2}\right]+e_{2}+e_{3}=w_{2}+e_{2}+e_{3} \\
& b_{3}=\left[\frac{n}{6}\right]-a_{3}=\left[\frac{z_{3}}{2}\right]+\left[\frac{z_{4}}{2}\right]=w_{3}, \\
& b_{4}=\left[\frac{n}{6}\right]-a_{4}=\left[\frac{z_{4}}{2}\right]+\left[\frac{z_{5}}{2}\right]+e_{4}=w_{4}+e_{4}, \\
& b_{5}=\left[\frac{n}{3}\right]-a_{5}=\left[\frac{z_{5}}{2}\right]+\left[\frac{z_{1}}{2}\right]+e_{5}+e_{1}=w_{5}+e_{5}+e_{1} .
\end{aligned}
$$

Отсюда, учитывая очевидное тождество $z_{i}=2\left[z_{i} / 2\right]+e_{i}$, получаем

$$
\begin{aligned}
b \cdot(1,1,-1,1,-1) & =2\left[\frac{n}{6}\right]-\left[\frac{n}{3}\right]+a \cdot(-1,-1,1,-1,1) \\
& =w \cdot(1,1,-1,1,-1)+e_{2}+e_{3}+e_{4}-e_{1}-e_{5} \\
& =z_{2}+e_{3}+e_{4}-e_{1}-e_{5}
\end{aligned}
$$

где '.' обозначает стандартное скалярное произведение векторов. Итак, имеем

$$
z_{2}=e_{1}+e_{5}-e_{3}-e_{4}+\left(2\left[\frac{n}{6}\right]-\left[\frac{n}{3}\right]\right)+a \cdot(-1,-1,1,-1,1)
$$

Положим для краткости $E=e_{1}+e_{5}-e_{3}-e_{4}, N=2[n / 6]-[n / 3]$ и $A=a \cdot(-1,-1,1$, $-1,1)$. Таким образом, $z_{2}=E+N+A$. Отметим, что $E \leqslant 2, A \leqslant 2$, и если $n=6 p+r$, где $r$ - остаток от деления $n$ на 6 , то $N=0$ при $0 \leqslant r \leqslant 2$ и $N=-1$ при $3 \leqslant r \leqslant 5$. Так как длина каждого конца не меньше 2 , в частности, $z_{2} \geqslant 2$, получаем, что при $0 \leqslant r \leqslant 2$ должно быть $A \geqslant 0$, а при $3 \leqslant r \leqslant 5$ должно быть $A \geqslant 1$.

Далее запишем условие того, что сумма длин всех боковин равна $n-5$ :

$$
4\left[\frac{n}{6}\right]+\left[\frac{n}{3}\right]-\sum a_{i}=n-5 .
$$

Подставляя в полученное равенство выражение $n=6 p+r$, найдем

$$
\sum a_{i}=5-r \text { при } 0 \leqslant r \leqslant 2, \quad \sum a_{i}=6-r \text { при } 3 \leqslant r \leqslant 5 .
$$

Рассмотрим случаи разных остатков $r$.

Пусть $r=0$. Тогда $\sum a_{i}=5$, поэтому каждое $a_{i}$ равно 1 . Отсюда $A=-1$, противоречие.

Пусть $r=1$. Тогда $\sum a_{i}=4$, поэтому ровно одно из $a_{i}$ равно 0, и $A \leqslant 0$. Из условия $z_{2} \geqslant 2$ получаем $A=0$ и $E=2$, откуда, в частности, $e_{3}=e_{4}=0$. Поэтому ind $\left(B_{3}\right)=1$, и по предложению $2.9 a_{3}=0$. Отсюда $A=-2$, противоречие.

Пусть $r=2$. Тогда $\sum a_{i}=3$, поэтому ровно два из $a_{i}$ равны 0 , и так как $A \geqslant 0$, имеем $A=1$. Тогда $a_{5}=1$, значит, по предложению 2.10 ind $\left(B_{5}\right)=-1$. Отсюда вытекает, что $e_{1}=e_{5}=0$, поэтому $E \leqslant 0$. Значит, $z_{2} \leqslant 1$, противоречие. 
Пусть $r=3$. Тогда $\sum a_{i}=3$, поэтому ровно два из $a_{i}$ равны 0 , следовательно, $A=1, E=2$, откуда $e_{1}=e_{5}=1$ и $e_{3}=e_{4}=0$. Таким образом, $\operatorname{ind}\left(B_{5}\right)=1$. Однако, по предложению 2.9 при любом $i<5$ имеем ind $\left(B_{i}\right)= \pm 1$. Но в соответствии со следствием 3.3 индекс одной из боковин обязан равняться 0 , противоречие.

Пусть $r=4$. Тогда $\sum a_{i}=2$ и ровно два из $a_{i}$ равны 1 . Учитывая, что $A \geqslant 1$, получаем $A=2$. Последнее влечет, что $a_{3}=1$. Отсюда ind $\left(B_{3}\right)=-1$, поэтому $e_{3}=e_{4}=1$. Значит, $E \leqslant 0$. Вспоминая, что $N=-1$, имеем $z_{2} \leqslant 1$, противоречие.

Пусть $r=5$. Тогда $\sum a_{i}=1$ и ровно одно из $a_{i}$ равно 1 . Поэтому $A=1$ и $E=2$, откуда вытекает, что одно из $a_{3}$ и $a_{5}$ отлично от $0, e_{1}=e_{5}=1$ и $e_{3}=e_{4}=0$. Последние два условия дают ind $\left(B_{3}\right)=\operatorname{ind}\left(B_{5}\right)=1$, поэтому $a_{3}=a_{5}=0$, противоречие.

Предложение 3.1 доказано.

Перейдем к рассмотрению второго случая.

ПРЕДЛОЖЕНИЕ 3.2. В сделанных выше предположениях скелет $S$, в действительности, не мохет иметь RM-реализаиии.

ДокАЗАТЕЛьСтво. Отметим, что теперь из внутренней ячейки, пересекающейся по сторонам с концами $Z_{1}$ и $Z_{2}$, может выходить перегородка, которую мы обозначим через $L$. Обозначим через $l_{1}$ и $l_{2}$ ребра перегородки $L$, по которым $L$ крепится к оставшейся части скелета $S$.

Возникает два случая: $l_{1}, l_{2}$ параллельны и $l_{1}, l_{2}$ непараллельны (рис. 5$)$.
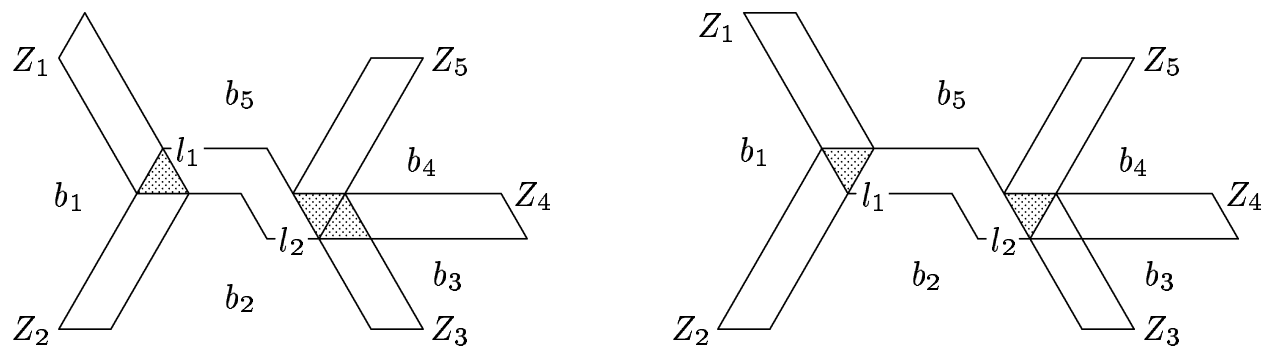

Рис. 5. Такие скелеты также не имеют RM-реализации

Пусть $l_{1}$ параллельно $l_{2}$. Легко видеть, что в этом случае длины боковин перегородки $L$ равны между собой независимо от формы перегородки. Добавив к введенным вьше обозначениям величину $x$, равную длине боковины перегородки $L$, получаем

$$
\begin{aligned}
& b_{1}=\left[\frac{n}{3}\right]-a_{1}=\left[\frac{z_{1}}{2}\right]+\left[\frac{z_{2}}{2}\right]+e_{1}=w_{1}+e_{1}, \\
& b_{2}=\left[\frac{n}{6}\right]-a_{2}=\left[\frac{z_{2}}{2}\right]+\left[\frac{z_{3}}{2}\right]+e_{2}+e_{3}+x=w_{2}+e_{2}+e_{3}+x, \\
& b_{3}=\left[\frac{n}{6}\right]-a_{3}=\left[\frac{z_{3}}{2}\right]+\left[\frac{z_{4}}{2}\right]=w_{3}, \\
& b_{4}=\left[\frac{n}{6}\right]-a_{4}=\left[\frac{z_{4}}{2}\right]+\left[\frac{z_{5}}{2}\right]+e_{4}=w_{4}+e_{4}, \\
& b_{5}=\left[\frac{n}{3}\right]-a_{5}=\left[\frac{z_{5}}{2}\right]+\left[\frac{z_{1}}{2}\right]+e_{5}+x=w_{5}+e_{5}+x .
\end{aligned}
$$


Отсюда вьводим, что

$$
\begin{aligned}
b \cdot(-1,1,-1,1,1) & =2\left[\frac{n}{6}\right]-\left[\frac{n}{3}\right]+a \cdot(1,-1,1,-1,-1) \\
& =w \cdot(-1,1,-1,1,1)+e_{5}+e_{2}+e_{3}+e_{4}+2 x-e_{1} \\
& =z_{5}+e_{2}+e_{3}+e_{4}+2 x-e_{1} .
\end{aligned}
$$

Итак, имеем

$$
z_{5}=e_{1}-e_{2}-e_{3}-e_{4}-2 x+\left(2\left[\frac{n}{6}\right]-\left[\frac{n}{3}\right]\right)+a \cdot(1,-1,1,-1,-1)
$$

Положим для краткости $E=e_{1}-e_{2}-e_{3}-e_{4}, N=2[n / 6]-[n / 3]$ и $A=a \cdot(1,-1,1,-1$, $-1)$. Таким образом, $z_{5}=E-2 x+N+A$. Отметим, что $E \leqslant 1, A \leqslant 2$, и если $n=6 p+r$, то $N=0$ при $0 \leqslant r \leqslant 2$ и $N=-1$ при $3 \leqslant r \leqslant 5$.

Легко видеть, что если $x>0$, то, очевидно, $z_{5} \leqslant 1$, противоречие. Поэтому $x=0$.

Далее, если $r \geqslant 3$, то $N=-1$, и из условия $z_{5} \geqslant 2$ вытекает, что $A=2$, поэтому $e_{1}=1, e_{2}=e_{3}=e_{4}=0$. Тогда $\operatorname{ind}\left(B_{2}\right)=-1$, поэтому $a_{2}=1$, значит $A \leqslant 1$, противоречие.

Итак, осталось рассмотреть случай $r<3$. Тогда по предложению 2.9 при $i>1$ имеем $a_{i}=0$ тогда и только тогда, когда ind $\left(B_{i}\right)=1$. Ясно, что условие $z_{5} \geqslant 2$ влечет $A \geqslant 1$, однако последнее неравенство вьполняется, если только, по крайней мере, два из трех $a_{2}, a_{4}$ и $a_{5}$ равны 0 , т.е., по крайней мере, две из трех боковин $B_{2}, B_{4}$ и $B_{5}$ имеют индекс 1 . Из леммы 3.2 получаем, что ind $\left(B_{2}\right)=1$, следовательно, $e_{2}=e_{3}=1$, откуда $E \leqslant-1$, и значит, $z_{5} \leqslant 1$, противоречие.

Случай параллельных $l_{1}$ и $l_{2}$ полностью разобран.

Пусть теперь $l_{1}$ непараллельно $l_{2}$. Легко видеть, что при выбранных обозначениях длина той боковины перегородки $L$, которая содержится в $B_{5}$, меньше на 1 , чем длина боковины из $L$, содержащейся в $B_{2}$. Обозначим через $x$ длину боковины $L \cap B_{5}$, тогда длина боковины $L \cap B_{2}$ равна $x+1$. Имеем

$$
\begin{aligned}
& b_{1}=\left[\frac{n}{3}\right]-a_{1}=\left[\frac{z_{1}}{2}\right]+\left[\frac{z_{2}}{2}\right]+e_{2}=w_{1}+e_{2}, \\
& b_{2}=\left[\frac{n}{6}\right]-a_{2}=\left[\frac{z_{2}}{2}\right]+\left[\frac{z_{3}}{2}\right]+e_{3}+x+1=w_{2}+e_{3}+x+1, \\
& b_{3}=\left[\frac{n}{6}\right]-a_{3}=\left[\frac{z_{3}}{2}\right]+\left[\frac{z_{4}}{2}\right]=w_{3}, \\
& b_{4}=\left[\frac{n}{6}\right]-a_{4}=\left[\frac{z_{4}}{2}\right]+\left[\frac{z_{5}}{2}\right]+e_{4}=w_{4}+e_{4}, \\
& b_{5}=\left[\frac{n}{3}\right]-a_{5}=\left[\frac{z_{5}}{2}\right]+\left[\frac{z_{1}}{2}\right]+e_{5}+e_{1}+x=w_{5}+e_{5}+e_{1}+x .
\end{aligned}
$$

Отсюда вьводим, что

$$
\begin{aligned}
b \cdot(-1,1,-1,1,1) & =2\left[\frac{n}{6}\right]-\left[\frac{n}{3}\right]+a \cdot(1,-1,1,-1,-1) \\
& =w \cdot(-1,1,-1,1,1)+e_{5}+e_{1}+e_{3}+e_{4}+2 x+1-e_{2} \\
& =z_{5}+e_{1}+e_{3}+e_{4}+2 x+1-e_{2} .
\end{aligned}
$$


Итак, имеем

$$
z_{5}=e_{2}-e_{1}-e_{3}-e_{4}-2 x-1+N+A,
$$

где $N=2[n / 6]-[n / 3]$ и $A=a \cdot(1,-1,1,-1,-1)$. Отметим, что $A \leqslant 2$ и $N \leqslant 0$.

Из условия $z_{5} \geqslant 2$ вытекает, что $A=2$, однако, тогда $a_{3}=1$, поэтому ind $\left(B_{3}\right) \neq 1$, значит, одно из $e_{3}$ и $e_{4}$ равно 1 , следовательно, $z_{5} \leqslant 1$, противоречие.

Случай непараллельных $l_{1}$ и $l_{2}$ полностью разобран. Тем самьм, предложение 3.2 полностью доказано.

Теперь доказательство теоремы 3.1 получается объединением доказательств предложений 3.1 и 3.2 .

Московский государственный университет

Поступило

им. М. В. Ломоносова

E-mail: tuz@difgeo.math.msu.su

Исправленный вариант

03.03.97

\section{СПИСОК ЦИТИРОВАННОЙ ЛИТЕРАТУРЫ}

[1] Ivanov A. O., Tuzhilin A. A. The Steiner problem for convex boundaries. II. The regular case // Adv. Soviet Math. 1993. V. 15. P. 93-131.

[2] Тужилин А. А. Минимальные бинарные деревья с правильной границей: случай скелетов с четырьмя концами // Матем. сб. 1996. Т. 187. № 4. С. 117-159.

[3] Hwang F. K., Richards D., Winter P. The Steiner Tree Problem. Amsterdam: Elsevier Science Publ. (to appear).

[4] Jarnik V., Kössler M. O minimalnich grafeth obeahujicich n danijch bodu // Cas. Pest. Mat. Fys. 1934. V. 63. P. 223-235.

[5] Melzak Z. A. On the problem of Steiner // Canad. Math. Bull. 1960. V. 4. P. 143-148.

[6] Garey M. R., Graham R. L., Johnson D.S. Some NP-complete geometric problems // 8th Annual ACM Symposium on Theory of Computating. New York: Assoc. Comput. Mach., 1976. P. 10-22.

[7] Preparata F., Shamos M. Computational geometry. An introduction. New York: Springer Verlag, 1985.

[8] Иванов А. О., Тужилин А. А. Решение задачи Штейнера для выпуклых границ // УМН. 1990. T. 45. № 2. C. 207-208.

[9] Иванов А. О., Тужилин А. А. Задача Штейнера для выпуклых границ или плоские минимальные сети // Матем. сб. 1991. Т. 182. № 12. С. 1813-1844.

[10] Иванов А. О., Тужилин А. А. Геометрия минимальных сетей и одномерная проблема Плато // УМН. 1992. Т. 47. №2(284). С. 53-115.

[11] Ivanov A. O., Tuzhilin A. A. The Steiner problem for convex boundaries. I. General case // Adv. Soviet Math. 1993. V. 15. P. 15-92.

[12] Ivanov A. O., Tuzhilin A. A. Minimal networks. The Steiner problem and its generalizations. N. W., Boca Raton, Florida: CRC Press, 1994.

[13] Иванов А.О., Тужилин А. А. Топологии локально минимальных плоских бинарных деревьев // УМН. 1994. Т. 49. №6. С. 191-192.

[14] Иванов А. О., Тужилин А. А. Взвешенные минимальные 2-деревья // УМН. 1995. T. 50 . № 3. C. $155-156$. 